\title{
Evaluating Mixture Adsorption Models Using Molecular Simulation
}

\author{
Joseph A. Swisher, Li-Chiang Lin, and Jihan Kim \\ Dept. of Chemical and Biomolecular Engineering, University of California, Berkeley, CA 94720 \\ Materials Sciences Div., Lawrence Berkeley National Laboratory, Berkeley, CA 94720 \\ Berend Smit \\ Dept. of Chemistry, University of California, Berkeley, CA 94720 \\ Materials Sciences Div., Lawrence Berkeley National Laboratory, Berkeley, CA 94720
}

DOI 10.1002/aic.14058

Published online March 7, 2013 in Wiley Online Library (wileyonlinelibrary.com)

\begin{abstract}
The design of adsorption-based separation processes using novel adsorbents requires reliable data for the adsorption of fluid mixtures on candidate adsorbents. Due to the difficulty of generating sufficient data across possible operating conditions, process designs generally rely on interpolation of pure-component data using a model, most commonly ideal adsorbed solution theory (IAST), and related theories. There are many cases where IAST fails to provide an adequate description of mixture adsorption, usually due to the fact that practical adsorbents do not have uniform surfaces. We have evaluated the use of a segregated version of IAST, where competition is assumed to occur at isolated adsorption sites. This simple modification can provide the correct description of adsorption across a large range of pressures using ideal isotherm models. We also demonstrate the importance of identifying multiple sites even for weakly adsorbing components to provide the correct behavior at high pressure. (C) 2013 American Institute of Chemical Engineers AIChE J, 59: 3054-3064, 2013
\end{abstract}

Keywords: adsorption/gas, simulation, molecular, computer simulations (MC and MD), thermodynamics/classical, zeolites

\section{Introduction}

Separating fluid mixtures via adsorption onto porous materials is a common process technology used in a wide variety of industrial contexts, including the separation of air, scrubbing solvent vapors from exhaust, and separation of xylene isomers. ${ }^{1}$ The design of adsorption-based processes requires reliable thermodynamic data for the fluid-adsorbent system over a range of potential process conditions. Collecting these data for the adsorption of mixtures of gases are particularly challenging due to the complexity of the experiments and interpretation of the resulting data. This difficulty is mitigated in practice by the use of a model, such as ideal adsorbed solution theory (IAST), to interpolate mixture data from pure-component data, which is simpler to collect. $^{2}$ Although IAST has been shown to provide good predictions for a wide variety of fluid-adsorbent systems, there are numerous cases where it has been shown that the predictions are inaccurate. ${ }^{3-10}$ The IAST model assumes ideal behavior of the adsorbed phase and that all components have access to the same uniform surface. This second assumption is \footnotetext{
Ad
article.

Additional Supporting Information may be found in the online version of this

Correspondence concerning this article should be addressed to B. Smit at berend-smit@berkeley.edu.
}

(C) 2013 American Institute of Chemical Engineers frequently violated in practical adsorbents, and variety of approaches have been proposed to correct for the inaccuracies it introduces, which will be discussed in more detail later. ${ }^{3,6-8,10-18}$ Recent efforts in synthesizing and predicting new materials have led to a plethora of potential new adsorbents, including metal-organic frameworks (MOFs), zeolitic imidazolate frameworks (ZIFs), and novel zeolites. These materials are very promising for several important gas separation applications. ${ }^{19}$ It is possible to introduce specific sites in these materials that selectively adsorb one of the components in preference to the others giving them a heterogeneous pore space by definition. From a practical point of view, it is important to reliably predict the mixture adsorption isotherms. This requires a better understanding how the structure of these materials influences the selection of the correct model for the adsorption of mixtures.

The large number of new porous materials that have been demonstrated in recent years introduces the practical problem of evaluating such a large number of materials for a given separation. For example, Deem and coworkers ${ }^{20}$ have developed a database of millions of hypothetical zeolite structures. Efforts by many researchers have revealed a wide array of MOFs with diverse structures and compositions. ${ }^{21}$ The nature of MOF chemistry suggests that there are potentially millions of materials when taking into account all possible combinations of metal nodes and organic linker molecules. ${ }^{19,22-24}$ When organized into databases, there is 
the possibility of screening very large sets of materials simultaneously to find those best suited for a particular application. ${ }^{18,21,25}$

An active area of research is the development of highthroughput screening techniques to identify the most promising materials for desired gas separations. In the absence of sufficient experimental data, direct computer simulations in the grand canonical ensemble ${ }^{26}$ can provide the data over a wide range of conditions for microporous materials like zeolites, ${ }^{27} \mathrm{MOFs},{ }^{28}$ and ZIFs. ${ }^{29}$ Both the experimental and the computational approaches focus on obtaining the pure-component isotherms and rely on IAST to accurately predict the mixture isotherms.

In this work, we develop a systematic approach to incorporate these chemical inhomogeneities in the IAST. This approach is based on the simple notion that one should divide the pore space into different regions and apply IAST on each region separately. Interesting, variations of this idea have appeared several times in the literature but, as we will demonstrate in this work, it can only be used successfully with a molecular understanding of the adsorption behavior of the pure-component isotherms. We illustrate with several practical examples that incomplete information about the pure-component isotherms, namely data at insufficiently high pressures where weakly adsorbing components saturate adsorption sites, can explain the problems associated with typical application of IAST.

At this point, we emphasize that we rely on molecular simulations to provide us with accurate data on both the pure components and the mixture isotherms. From a purely conceptional point of view, there is no fundamental difference for IAST, in predicting mixture isotherms, whether these pure-component data are obtained from molecular simulations or experiments. Of course, only if our simulations are representative for the experimental system, our conclusions also hold for the experimental conditions.

One of the main conclusions of this work is that a reliable prediction of the mixture isotherm requires knowledge on the number of adsorption site and which component preferentially adsorbs on which site. For many systems, this can be reliably obtained from the pure-component isotherms. However, in some, and usually the most interesting, cases this molecular information is lacking. We also demonstrate how molecular simulation can be used to address these questions. Given that the assumption of a uniform surface is often violated in practice, simulations provide a molecule-scale picture that can be used to suggest the siting of molecules in the adsorbent's pores. These strong sites have important implications for the best model to interpolate the data. Simulations can also predict adsorption at very high pressures that may be infeasible in practice. Accurate information about the saturation loading of weakly adsorbed components may only be available at these high pressures and can help correctly parameterize the pure-component isotherms.

\section{Adsorbed Solution Theories}

\section{IAST}

Due to the importance of making reliable predictions of adsorption isotherms for fluid mixtures, many models have been proposed that attempt to predict the adsorbed phase composition and loading based on pure-component data and, in some models, properties of the fluid mixture. Most practical models rely on some variant of the IAST of Myers and Prausnitz. $^{2}$ This approach is popular due to the fact that it relies solely on the isotherms of the pure components in the fluid mixture and is amenable to rapid solution using numerical techniques. IAST has been used extensively to describe adsorption from multicomponent mixtures in zeolites and other adsorbents, including mixtures of alkanes, $\mathrm{CO}_{2}, \mathrm{CH}_{4}$, and $\mathrm{N}_{2} \cdot{ }^{30-33}$ Extensions to IAST include the real adsorbed solution theory ${ }^{3}$ and the non-ideal AST, ${ }^{11}$ which attempts to account for nonideal behavior of the adsorbed phase by correlating activity coefficients from binary adsorption data or assumptions about the distribution of sites, respectively. Other approaches have attempted to deal with problems surrounding the assumption of a perfectly uniform surface. One key modification these approaches make to IAST is that the surface is assumed to be composed of independent adsorption sites, and any mixture model should be applied separately for each site. For the sake of clarity, this concept will be called segregated IAST (SIAST) when applied in the context of this work, and the terminology of other authors will be adopted when addressing their work.

The concept of modeling adsorption as independent processes at distinct sites or volumes has been applied many times before to improve mixture adsorption predictions relative to IAST. Valenzuela et al. ${ }^{12}$ proposed what they termed heterogeneous IAST where each component is assumed to have a distribution of adsorption sites characterized by an adsorption energy. IAST is applied to each site separately based on a fitted energy difference between sites. Moon and Tien ${ }^{6}$ took the analysis a step further and proposed that matching the sites of different adsorbates based on a site-matching criteria that accounts for correlation of the adsorption of different components on the same site. Eiden and Schlünder ${ }^{7,8}$ applied a similar approach to the analysis of dichloromethane and benzene adsorption on activated carbons. Calling the approach multiphase IAST, they assumed a pore filling mechanism and applied IAST to separate sites based on a difference in saturation volumes between components. Cerofolini and Rudznski ${ }^{13}$ categorized these approaches as patchwise theories, as they treat the surface of the adsorbent material as composed of a set of discrete areas or volumes where a model was applied to each individual patch, but there was no interaction between different patches. One drawback of these approaches from a practical standpoint is that they require additional assumptions about the system, usually in the form of the distribution site energies. These distributions must also be parameterized, which can be difficult given the nature of the inverse problem. ${ }^{14}$ Recent work by Ritter et al. ${ }^{15}$ attempted to avoid this issue using a sum of competitive Langmuir sites approach, which they term a dual-process Langmuir (DPL) isotherm, using the parameters from fitting a dual-site Langmuir isotherm to the pure-component data. Their approach also permits accounting for nonideality in the system by applying the sitematching approach of Moon and Tien by simply swapping the parameters of a particular component between terms of the isotherm. Unfortunately, there is no clear method for deciding when this switch is appropriate other than comparing with experimental mixture data. A disadvantage of these approaches is that they potentially require a large quantity of detailed adsorption data at a variety of conditions that may not be easy or cheap to generate and often are specific to the material being studied.

In more recent work, several authors have attempted to use molecular simulations to evaluate the usefulness of IAST and similar approaches for predicting mixture properties in zeolites and MOFs. Cessford et al. ${ }^{16}$ have provided a comprehensive overview of the topic in their recent article, and 
we will only address a few highlights here. Krishna and coworkers $^{34}$ have evaluated the use of IAST to predict mixtures of alkanes in the zeolite MFI and $\mathrm{CO}_{2}: \mathrm{CH}_{4}$ mixtures in cage-type zeolites. ${ }^{10}$ In this latter article, the authors observed that IAST failed to give a good description of the mixture at higher pressures and ascribed this to a segregation of the two components due to preferential adsorption at either the center of the cages or the windows between cages. Jee and Sholl ${ }^{17}$ suggested that using a segregated model for $\mathrm{CO}_{2}: \mathrm{CH}_{4}$ adsorption in zeolite DDR improves predicted isotherms relative to IAST, but is still inaccurate at high total fugacity. Van Heest et al. ${ }^{18}$ performed an extensive screening of MOFs for separating noble gas mixtures and noted that accounting for regions that would be inaccessible to one component of the mixture was important for correctly predicting adsorption selectivity. A key aspect of these studies is that they were able to correlate specific structural features of the adsorbents to the inaccuracy of IAST.

\section{SIAST}

While the IAST model does not rely on a specific description of the molecular system, it is usually interpreted in terms of a hypothetical uniform surface for the adsorption of the components of the mixture. Due to the atomic scale heterogeneity of nanoporous materials, it is useful to view the materials as having multiple "surfaces" where molecules can adsorb. Each of these surfaces can have a separate equilibrium, which we assume can be described with an ideal model. Strictly speaking, for microporous adsorbents like zeolites, the concept of spreading pressure is not applicable, because there is no well-defined surface. ${ }^{11,35}$ However, the idea of accessible surface area or pore volume is still a useful concept for diagnosing failures of IAST. If the components of the mixture can access different parts of the material, one component may be able to diffuse into cavities not accessible to other components and is, thus, no longer competing with the other components for the same adsorption sites. In recent work on the screening of zeolites for the separation of $\mathrm{CO}_{2}$ and $\mathrm{N}_{2}$, we discovered a class of materials that had particularly strong adsorption sites for $\mathrm{CO}_{2} \cdot{ }^{25}$ These were often small pockets that could accommodate a single $\mathrm{CO}_{2}$ molecule, and the arrangement of the framework atoms around the pocket provides a site with a particularly favorable interaction energy for the molecule. Although $\mathrm{N}_{2}$ is not formally excluded from these pockets, they are more likely to be occupied by $\mathrm{CO}_{2}$ when the two gases are adsorbing competitively. Materials of this sort typically exhibit an isotherm with a plateau or step at intermediate loading that can be accurately correlated with the dual-site Langmuir isotherm equation. The loading at the plateau corresponds to the density of strong adsorption sites in the unit cell.

To more accurately describe the total loading of a system that may have spatially distinct adsorption sites, we propose that applying IAST to distinct sites can provide more accurate predictions in many cases. Instead of considering the available pore volume to be one continuous space, the volume may be subdivided into natural regions where separate competition processes take place. For materials where it is possible to identify segregated sites, it is possible to model the mixture adsorption as the sum of separate competitions, which in turn may be modeled using IAST. Figure 1 shows how this might be extended to an arbitrary number of adsorbed phases. Equation 1 relates the isotherm of component $i$ at site $j$ to the surface potential.

$$
\psi_{i j}=\int_{0}^{f_{i j}^{\circ}} n_{i j}(f) d \ln f
$$

In Eq. 1, the surface potential for adsorption of component $i$ at site $j$ is related to an adsorption isotherm representing only site $j$. In this work, we will assume that each site can be described by a single-site Langmuir isotherm

$$
n_{i j}(f)=\frac{m_{i j} b_{i j} f}{1+b_{i j} f}
$$

giving the following expression for the surface potential

$$
\psi_{i j}\left(f_{i j}^{\circ}\right)=m_{i j} \log \left(1+b_{i j} f_{i j}^{\circ}\right)
$$

where $m_{i j}$ and $b_{i j}$ are the saturation loading and Langmuir constant for adsorption, respectively, of component $i$ at site $j$. The condition for equilibrium may be expressed as Equation 4 for competitive adsorption between $N$ components at site $j$.

$$
\psi_{1 j}=\psi_{2 j}=\ldots=\psi_{N j}
$$

As we treat the adsorbed phase as composed of independent phases, each is related to the gas phase independently.

$$
f y_{i}=x_{i j} f_{i j}^{\circ}\left(\psi_{i j}\right)
$$

There is an independent mass balance on each phase.

$$
\sum_{i=1}^{N} x_{i j}=1
$$

The total loading in phase $j$ is given by

$$
n_{t j}=\left[\sum_{i=1}^{N} \frac{x_{i j}}{n_{i j}\left(P_{i j}^{\circ}\right)}\right]^{-1}
$$

and loading of component $i$ in phase $j$ is given by

$$
n_{i j}=x_{i j} n_{t j}
$$

The total loading of component $i$ is simply the sum of the loadings in the individual phases.

$$
n_{i}=\sum_{j=1}^{M} n_{i j}
$$

While this method is general, experimental isotherms may not cover a large enough range of pressures to accurately determine the saturation loadings for all components at each site. We are also neglecting any sort of accounting for the nonideality of the absorbed mixture that may exist at high density. Except at the highest densities, the interactions felt by molecules in nanoporous materials are dominated by the atoms of the material framework any contribution to nonideality from the components themselves is likely small in comparison.

To compare traditional IAST with the current approach, the dual-site Langmuir isotherm model was fitted to the purecomponent isotherms using nonlinear least-squares regression.

$$
n_{i}(f)=\frac{m_{i 1} b_{i 1} f}{1+b_{i 1} f}+\frac{m_{i 2} b_{i 2} f}{1+b_{i 2} f}
$$

For materials with strongly segregated sites for adsorption, the saturation at the first site was fixed to the same value for 


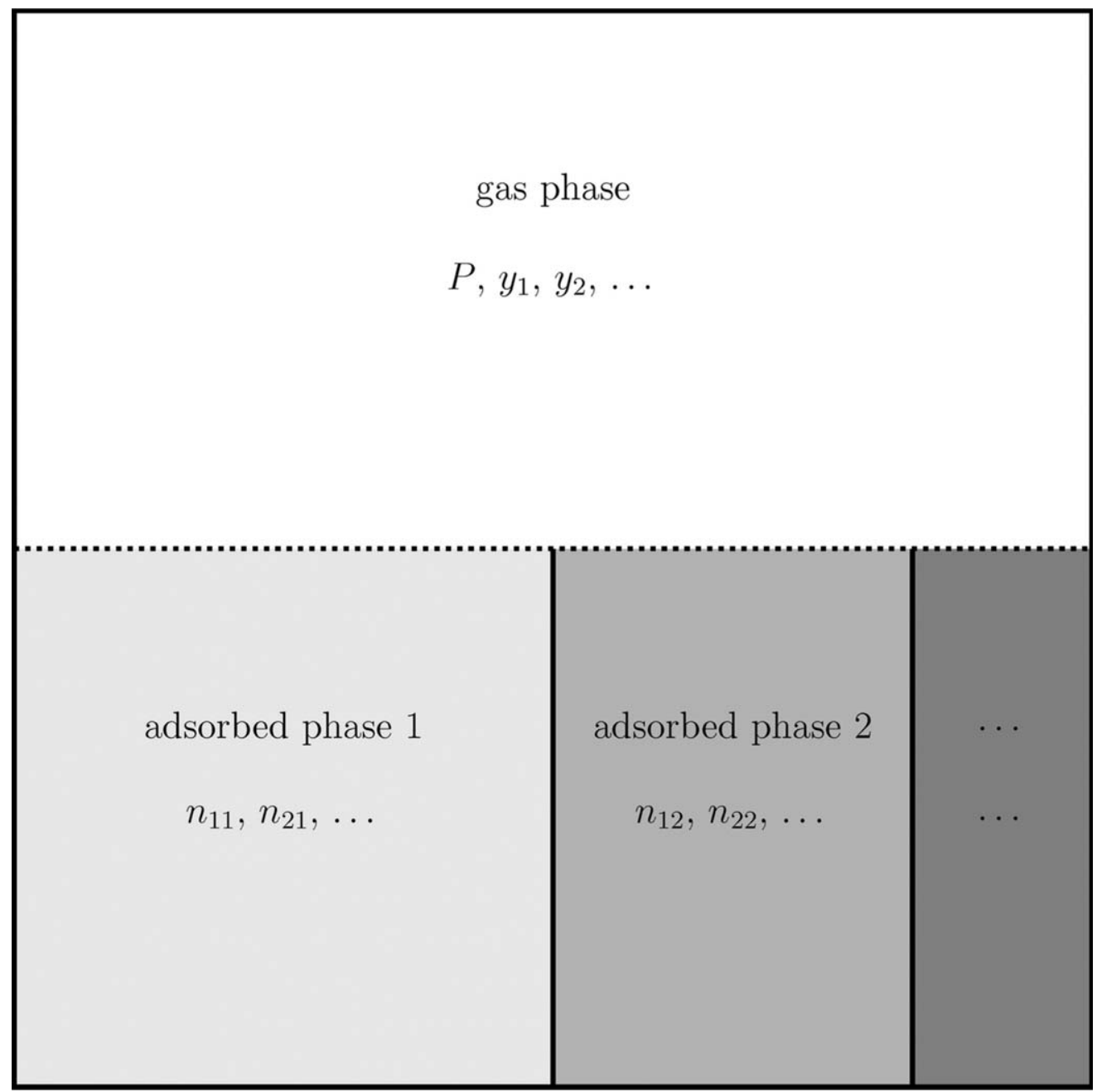

Figure 1. Schematic diagram of the phases in the SIAST model.

A gas phase (white) is in equilibrium with $M$ independent adsorbed phases (shades of gray). The whole system is at a constant temperature. The gas phase is characterized by a total pressure $\boldsymbol{P}$ and its composition, usually expressed as the mole fraction of all components $y_{i}$. Each adsorbed $j$ phase is characterized by the loading of each component $i$, $n_{i j}$ in equilibrium with the gas phase. Each adsorbed phase is in equilibrium separately with the gas phase.

both $\mathrm{CO}_{2}$ and $\mathrm{N}_{2}$, reflecting the fact that only one molecule of any component can occupy one of these sites at a given time.

\section{Methods}

To investigate different models of adsorption, grand canonical Monte Carlo (GCMC) simulations were used to generate pure-component and mixture isotherms for mixtures of $\mathrm{CO}_{2}$ and $\mathrm{N}_{2}$ in different zeolites and $\mathrm{CO}_{2}$ and $\mathrm{C}_{3} \mathrm{H}_{8}$ in the zeolite MOR. These were treated as the reference data that allow us to compare the predictions of different models. Simulations in the grand canonical ensemble involve specifying the temperature and volume of an adsorbent system and the chemical potentials of all the adsorbing components. ${ }^{26}$ The simulation box was defined by a tiling of one or more crystallographic unit cells of a given material. All framework atoms were held fixed. Trial configurations were generated by attempting to move, rotate, reinsert, or remove an existing molecule or adding a new molecule to the simulation box. The energy of all interactions was described using the 12-6 Lennard-
Jones potential plus an electrostatic term. The Ewald summation technique was used for the electrostatic interactions. All short-range interactions were cut off and shifted to zero at $12.0 \AA$. Interaction parameters and partial atomic charges were taken from different sources, depending on the system under study. $\mathrm{CO}_{2}$ and $\mathrm{N}_{2}$ adsorption in siliceous zeolites were modeled using the parameters of García-Pérez et al. ${ }^{36} \mathrm{C}_{3} \mathrm{H}_{8}$ adsorption in MOR was parameterized using the force field of Dubbeldam et al. ${ }^{37}$ For simulations in aluminosilicate zeolites, $\mathrm{CO}_{2}$ and sodium interaction parameters were taken from García-Sánchez et al. $^{38} \mathrm{~N}_{2}$ interaction parameters in these materials were developed as a part of this work and are reported in the Supporting Information.

\section{Results and Discussion}

We have broken down the analysis into a three separate cases based on the degree of segregation between adsorption sites and the relative preference of each adsorbing component for each of the adsorption sites. In one extreme case of segregation, the adsorption sites may be spatially separated 

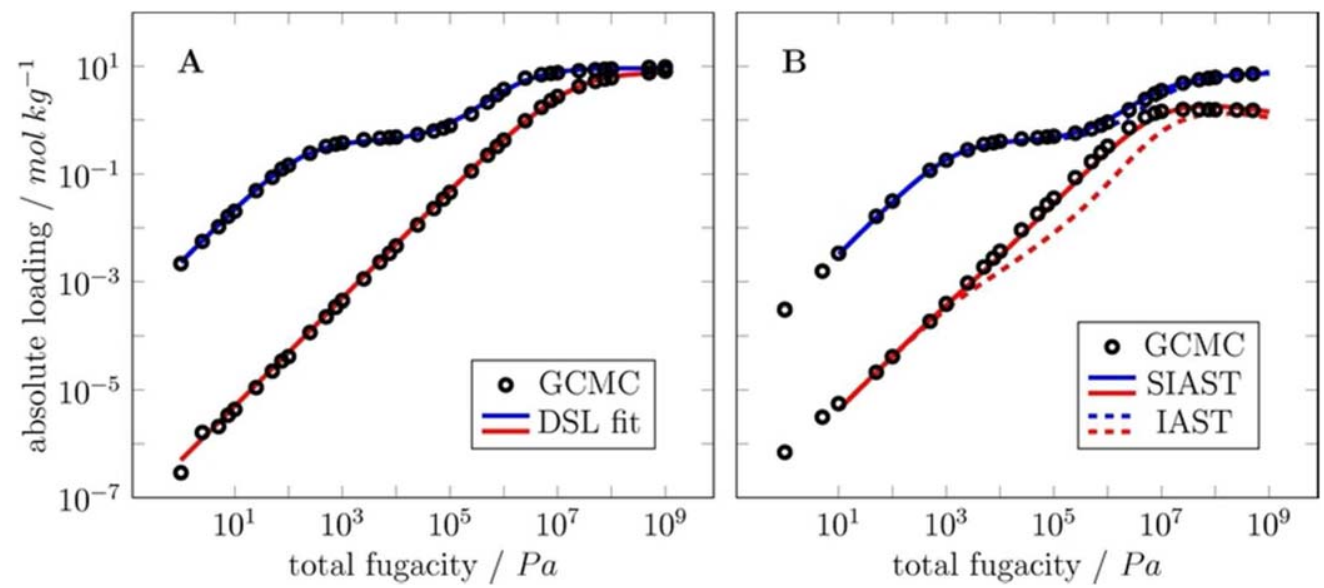

Figure 2. Comparison of isotherms computed via GCMC and different mixture adsorption models in pure silica PCOD8286959 at $300 \mathrm{~K}$.

(A) Pure-component isotherms of $\mathrm{CO}_{2}$ (blue) and $\mathrm{N}_{2}$ (red) with lines indicating the fit of the dual-site Langmuir model (Eq. 10). (B) Mixture adsorption isotherms for a $14 \% \mathrm{CO}_{2}$ (blue), $86 \% \mathrm{~N}_{2}$ mixture. Symbols indicate GCMC results, solid lines represent the SIAST model, and dashed lines represents the traditional IAST model. [Color figure can be viewed in the online issue, which is available at wileyonlinelibrary.com.]

from one another and diffusion between sites requiring crossing a barrier. Each of these sites can represent a different free energy for each component, and components will occupy the sites in the order that represents the lowest free energy for the component. In this work, we have considered materials with two distinct adsorption sites, and we distinguish whether a given mixture exhibits a normal site preference, that is, components occupy sites in the same order, or reverse site preference, where one component occupies sites in the reverse order that the other component does. This parallels the site correlation concept used by Moon and Tien $^{6}$ and Ritter et al. ${ }^{15}$ We also consider the case where sites are not isolated from one another and evaluate the use of competitive isotherm models.

\section{Materials with isolated sites, normal site preference}

GCMC simulations were performed for pure $\mathrm{CO}_{2}$ and pure $\mathrm{N}_{2}$ in hypothetical zeolites PCOD8286959 and PCOD8200029 from Deem's database of hypothetical materials $^{20}$ at $300 \mathrm{~K}$. These pure-component isotherms are shown in Figures 2A and 4A, respectively. The isotherms were simulated from very low fugacity (on the order of $10 \mathrm{~Pa}$ ) up to $10^{9} \mathrm{~Pa}$ fugacity. The very high fugacities are required to ensure that the plateaus in the isotherm are accurately represented. In the case of the more weakly adsorbing $\mathrm{N}_{2}$, fugacities above $10^{7}$ and $10^{6} \mathrm{~Pa}$ for PCOD8286959 and PCOD8200029, respectively, were required for the isotherm to approach a saturation loading. All pure-component isotherms were fitted using the dual-site Langmuir isotherm (Eq. 10) with the constraint that the saturation loading of the first adsorption site $\left(m_{i 1}\right)$ was fixed to be identical for both $\mathrm{CO}_{2}$ and $\mathrm{N}_{2}$ (fitted parameters can be found in the Supporting Information). Previous work ${ }^{25}$ has indicated that adsorption in these materials occurs first at small localized pockets in the material that can accommodate only a single molecule. Figures 3 and 5 show accumulated snapshots of $\mathrm{CO}_{2}$ and $\mathrm{N}_{2}$ locations in PCOD8286959 and PCOD8200029, respectively, taken from the GCMC simulations of the mixture at $10^{9} \mathrm{~Pa}$. In PCOD8286959 (Figure 3), large main channels (purple circle) run parallel to the $c$ crystal axis, and small pockets (orange circle) connect between channels in the $b$ direction. The density of these sites is set by the crystal structure of the material and hence should be identical for both components. Although $\mathrm{N}_{2}$ does not exhibit a pronounced step in the isotherm, because it does not have as strong a preference for this site as $\mathrm{CO}_{2}$ does, this constraint is required to ensure the correct description of the total loading of each site in the mixture model. In PCOD8286959 (Figure 3), the molecules can adsorb in two separate pore systems, one running parallel the $c$ crystal axis (orange circle and arrow) and the other running parallel to the $b$ axis (purple circle and arrow). The sizes of these two channels are slightly different, with $\mathrm{CO}_{2}$ preferring to adsorb in the channels parallel to the $b$ axis, as shown by the predominance of blue points in Figure 3.

Figures $2 \mathrm{~B}$ and $4 \mathrm{~B}$ show adsorption isotherms for a $14 \%$ $\mathrm{CO}_{2}, 86 \% \mathrm{~N}_{2}$ mixture at $300 \mathrm{~K}$ predicted using GCMC, the SIAST model, and the original IAST model in PCOD8286959 and PCOD8200029, respectively. The original IAST data were generated by integrating Eq. 1 directly with Eq. 10 for the two components. For both materials, the models both agree with the GCMC data at very low fugacity. At these low fugacities, adsorption is occurring in the linear or Henry's law regime of the isotherm. Each component is in effect adsorbing independently and is completely described by its Henry coefficient. Both the segregated and traditional IAST model agree up to fugacities commensurate with the first saturation loading in the pure $\mathrm{CO}_{2}$ isotherm. Above these fugacities, the $\mathrm{N}_{2}$ loading predicted by traditional IAST is lower than the GCMC results. In the case of PCOD8286959 (Figure 2B), the IAST-predicted $\mathrm{N}_{2}$ loading matches the GCMC results at fugacities above $10^{7} \mathrm{~Pa}$, whereas in PCOD8200029, the IAST-predicted $\mathrm{N}_{2}$ loading is consistently 10 times lower than the GCMC isotherm. For both of these materials, the SIAST model provides excellent agreement with the GCMC results at all fugacities.

The effect of using the traditional IAST model is that the step in the $\mathrm{CO}_{2}$ isotherm induces an effective step in the $\mathrm{N}_{2}$ isotherm when using IAST, because it imposes a competitive adsorption process between $\mathrm{CO}_{2}$ adsorbing at the stronger adsorption sites and $\mathrm{N}_{2}$ adsorbing at the weaker adsorption sites. The adsorption of $\mathrm{N}_{2}$ at the weaker sites in the 


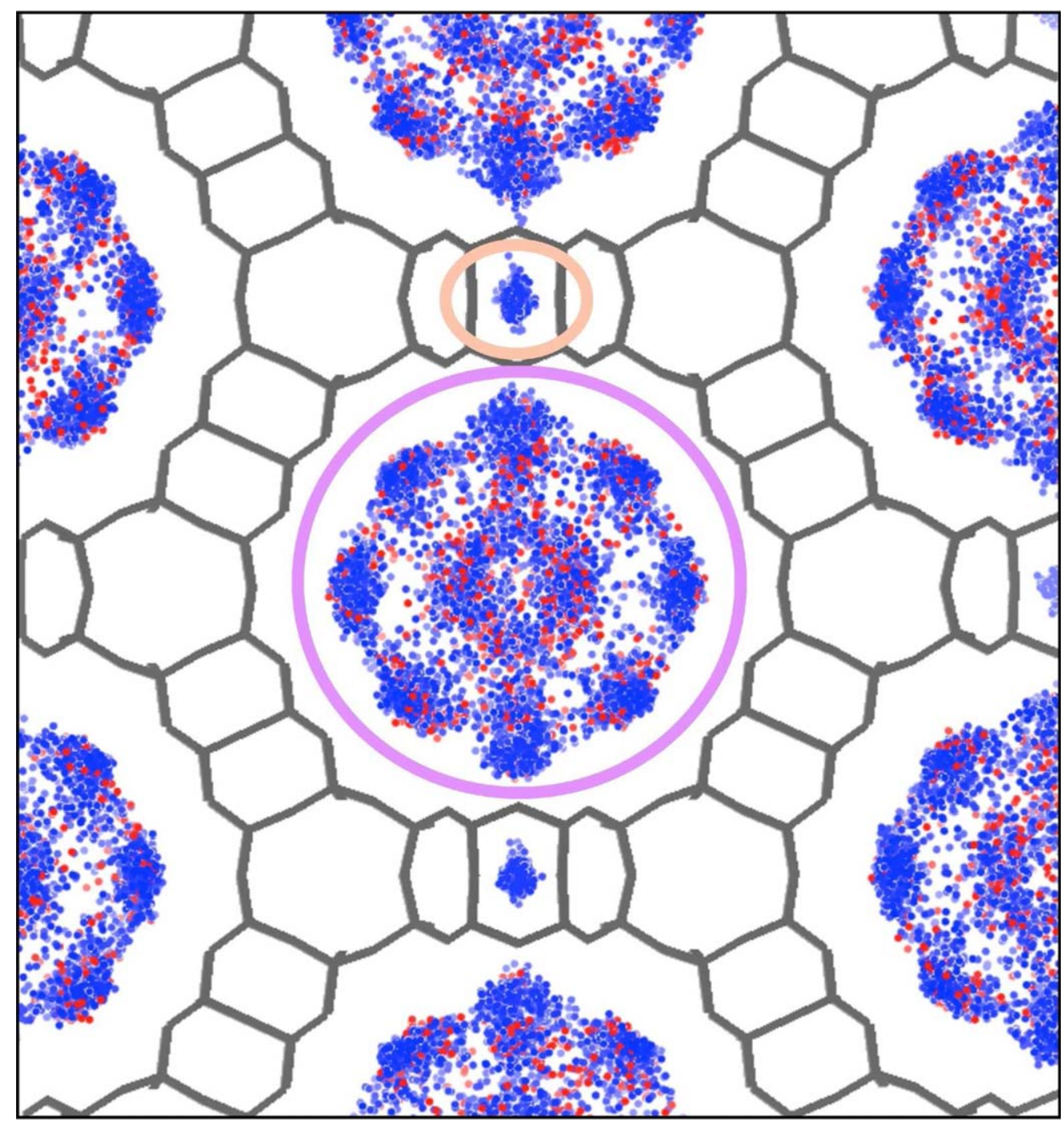

Figure 3. Snapshots of $\mathrm{CO}_{2}$ (blue) and $\mathrm{N}_{2}$ (red) center-of-mass configurations in zeolite PCOD8286959 during simulation at $10_{9}$ Pa fugacity and $300 \mathrm{~K}$.

Framework silicon and oxygen atoms are drawn in dark gray, and the centers-of-mass were collected from 10,000 samples during the GCMC simulation. The purple and orange circles indicate the main channels and the small pockets of the material, respectively. Due to the affinity of $\mathrm{CO}_{2}$ for the small pocket, $\mathrm{N}_{2}$ is effectively excluded and only adsorbs in the main channel. [Color figure can be viewed in the online issue, which is available at wileyonlinelibrary.com.]

framework can occur simultaneously and independently of adsorption at the stronger sites due to the fact that there is likely a barrier between adsorption at these distinct sites. The fact that $\mathrm{CO}_{2}$ is more likely to adsorb at the stronger sites first frees up the rest of the volume of the material where it would otherwise adsorb to provide adsorption sites for $\mathrm{N}_{2}$. We also note that the relative saturation loadings of the two adsorption sites do not appear to matter. If we compare the fitted isotherm parameters the case of PCOD8286959 (Figure 2), the first saturation loading is $0.4229 \mathrm{~mol} \mathrm{~kg}^{-1}$, compared to $8.811 \mathrm{~mol} \mathrm{~kg}^{-1}$ for the second saturation loading of $\mathrm{CO}_{2}$. The same values for the two sites in PCOD8200029 (Figure 4) are $1.510 \mathrm{~mol} \mathrm{~kg}^{-1}$ and $0.7354 \mathrm{~mol} \mathrm{~kg}^{-1}$, respectively. The first site accounts for $4.6 \%$ of the total saturation in PCOD8286959 but over $67 \%$ of the total saturation loading in PCOD8200029. Although one site in the material may provide the majority of all adsorption sites, it is important to partition them correctly, especially to predict mixture adsorption at high fugacity where both sites will likely be occupied to some extent.

\section{Materials with isolated sites, reverse site preference}

One set of data that has been used to evaluate different mixture adsorption models was the adsorption of $\mathrm{CO}_{2}: \mathrm{C}_{3} \mathrm{H}_{8}$ on H-MOR. Valenzuela et al., Moon and Tien, and Ritter et al. applied their approaches and showed that different segregated models improved predictions for the system relative to IAST. ${ }^{6,12,15} \mathrm{We}$ computed isotherms for pure $\mathrm{CO}_{2}$, pure $\mathrm{C}_{3} \mathrm{H}_{8}$ (Figure 6A) and a 50:50 $\mathrm{CO}_{2}: \mathrm{C}_{3} \mathrm{H}_{8}$ mixture (Figure $6 \mathrm{~B})$ adsorbing in the pure-silica form of MOR at $300 \mathrm{~K}$. The MOR topology has well-known side pockets along the main pores of the material, and this is reflected by the step in the isotherms for both components. Figure 7 shows center-ofmass configurations of $\mathrm{CO}_{2}$ and $\mathrm{C}_{3} \mathrm{H}_{8}$ recorded during GCMC simulations at low $\left(10^{3} \mathrm{~Pa}\right)$ and high $\left(10^{9} \mathrm{~Pa}\right)$ fugacity of both pure components and the mixture described earlier. The purple circle indicates the main channels of the material, running parallel to the $c$ crystal axis and the orange circles indicate the side pockets along these channels. As can be seen in the pure $\mathrm{CO}_{2}$ snapshots, $\mathrm{CO}_{2}$ is most likely to 

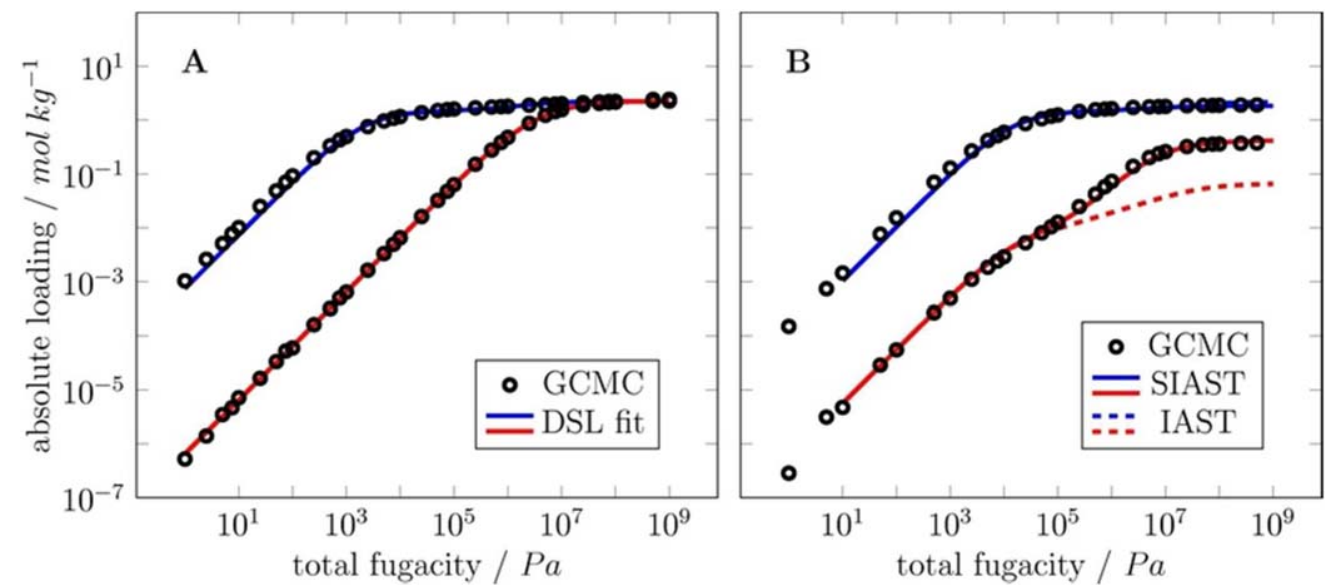

Figure 4. Comparison of isotherms computed via GCMC and different mixture adsorption models in pure silica PCOD8200029 at $300 \mathrm{~K}$.

(A) Pure-component isotherms of $\mathrm{CO}_{2}$ (blue) and $\mathrm{N}_{2}$ (red) with lines indicating the fit of the dual-site Langmuir model (Eq. 10). (B) Mixture adsorption isotherms for a $14 \% \mathrm{CO}_{2}$ (blue), $86 \% \mathrm{~N}_{2}$ mixture. Symbols indicate GCMC results, solid lines represent the SIAST model, and dashed lines represents the traditional IAST model. [Color figure can be viewed in the online issue, which is available at wileyonlinelibrary.com.]

be found in the side pockets at low fugacity, then as those are saturated, stars to fill the main channels. The reverse is true for $\mathrm{C}_{3} \mathrm{H}_{8}$. We note that the second saturation for $\mathrm{C}_{3} \mathrm{H}_{8}$ does not appear until the fugacity goes above $10^{8} \mathrm{~Pa}$, whereas $\mathrm{CO}_{2}$ begins to saturate its second site below $10^{6} \mathrm{~Pa}$ fugacity. As these components have opposite preferences, at low fugacity, $\mathrm{C}_{3} \mathrm{H}_{8}$ occupies the main channels whereas $\mathrm{CO}_{2}$ resides in the side pockets. At high fugacity, however, $\mathrm{CO}_{2}$ has saturated the side pockets and competes for the main channels with $\mathrm{C}_{3} \mathrm{H}_{8}$, which can be seen by the relatively few red points in the mixture snapshots for this condition.

Figure 6B compares the predictions of IAST, SIAST, and the DPL model with the results of the GCMC simulations. The dual-site Langmuir parameters for both pure components were fit without restriction. Although we did not constrain them as in the previous case, we observed that the first saturation loading for $\mathrm{CO}_{2}\left(4.165 \mathrm{~mol} \mathrm{~kg}^{-1}\right)$ was comparable to the second saturation loading for $\mathrm{C}_{3} \mathrm{H}_{8}\left(4.993 \mathrm{~mol} \mathrm{~kg}^{-1}\right)$. As shown by Ritter et al., reversing the sense of the two sites in the isotherms of $\mathrm{CO}_{2}$ and $\mathrm{C}_{3} \mathrm{H}_{8}$ provided the best predictions of experimental mixture compositions on $\mathrm{H}$ HOR. ${ }^{15}$ In our application of the SIAST and DPL models, we also made this switch, so that the first site in the $\mathrm{CO}_{2}$ isotherm competes with the second site in the $\mathrm{C}_{3} \mathrm{H}_{8}$ isotherm and vice versa. Traditional IAST does not provide for this reversal, as the entire isotherm is integrated simultaneously and provides the poorest description of the data. SIAST and the DPL model provide good agreement with the GCMC results at low fugacity. At high fugacity, the DPL model predicts a constant composition after the fugacity increases beyond $10^{6} \mathrm{~Pa}$. This is expected for competitive isotherm models, since the limit for this form of isotherm as fugacity or pressure approaches infinity is simply the saturation
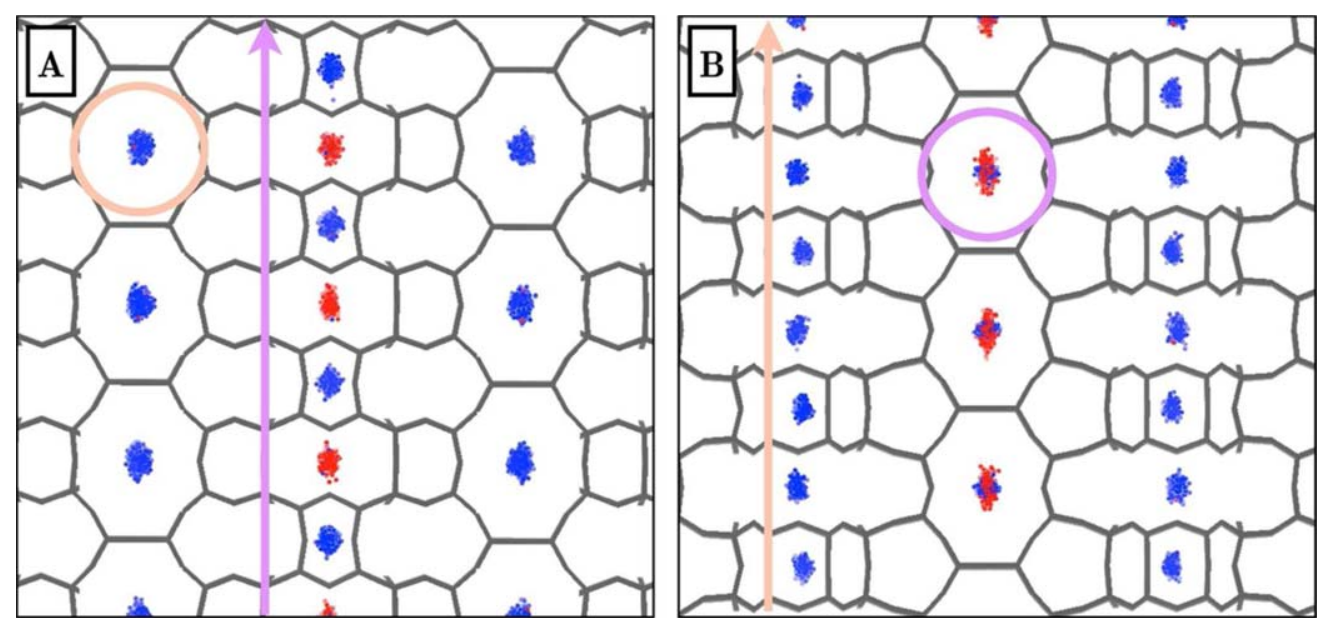

Figure 5. Snapshots of $\mathrm{CO}_{2}$ (blue) and $\mathrm{N}_{2}$ (red) center-of-mass configurations in zeolite PCOD8200029 during simulation at $10_{9}$ fugacity and $300 \mathrm{~K}$.

Framework silicon and oxygen atoms are drawn in dark gray, and the centers-of-mass were collected from 10,000 samples during the GCMC simulation. The pores of the material consist of two orthogonal pore systems, indicated by the purple and orange arrows and circles. (A) and (B) show the views down the $c$ and $b$ crystallographic axes, respectively. The pores are small enough in the channel indicated by orange that $\mathrm{CO}_{2}$ prefers to site there and tends to exclude $\mathrm{N}_{2}$. Both components are accommodated in the slightly larger channels indicated by purple. [Color figure can be viewed in the online issue, which is available at wileyonlinelibrary.com.] 

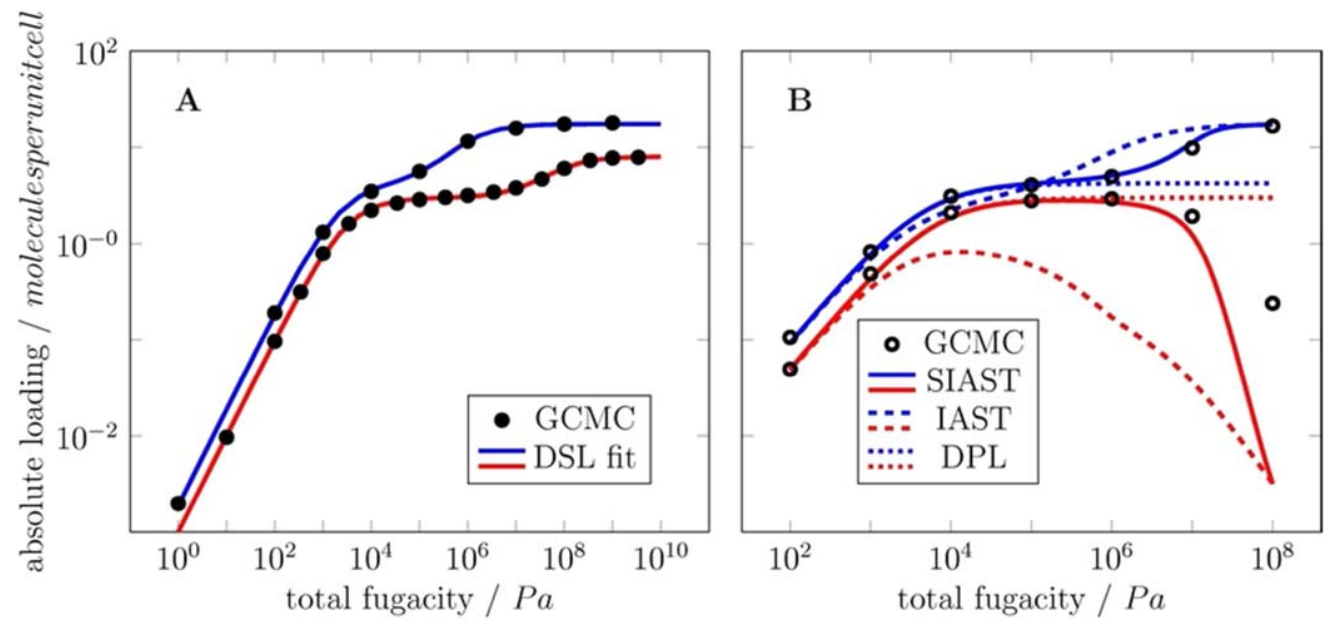

Figure 6. Comparison of isotherms computed via GCMC and different mixture adsorption models in pure silica MOR at $300 \mathrm{~K}$.

(A) Pure-component isotherms of $\mathrm{CO}_{2}$ (blue) and $\mathrm{C}_{3} \mathrm{H}_{8}$ (red) with lines indicating the fit of the dual-site Langmuir model (Eq. 10). (B) Mixture adsorption isotherms for a $50 \% \mathrm{CO}_{2}$ (blue), $50 \% \mathrm{C}_{3} \mathrm{H}_{8}$ mixture. Symbols indicate GCMC results, solid lines represent the SIAST model, dashed lines represents the traditional IAST model, and densely dotted lines are predictions of the DPL model of Ritter et al. ${ }^{15}$ [Color figure can be viewed in the online issue, which is available at wileyonlinelibrary.com.]

loading of the component. The GCMC simulations predict that the loading of $\mathrm{C}_{3} \mathrm{H}_{8}$ will eventually decrease. The replacement of $\mathrm{C}_{3} \mathrm{H}_{8}$ by $\mathrm{CO}_{2}$ in the pores should be expected at very high fugacity due to the entropic effect of smaller molecule $\mathrm{CO}_{2}$ filling the available volume more effectively than $\mathrm{C}_{3} \mathrm{H}_{8}$. The SIAST model provides a good fit to the GCMC results for $\mathrm{CO}_{2}$ over the entire fugacity range, and $\mathrm{C}_{3} \mathrm{H}_{8}$ is well described up to about $10^{7} \mathrm{~Pa}$ total fugacity. Above this fugacity, the predicted $\mathrm{C}_{3} \mathrm{H}_{8}$ loading decreases more rapidly than observed via GCMC. This may be due to other features in the isotherm that would be observed by simulating the pure components at higher fugacity then we did and would require an additional site in the isotherm to describe.

The SIAST and DPL model required that the second site in the $\mathrm{C}_{3} \mathrm{H}_{8}$ isotherm was matched with the first site of the $\mathrm{CO}_{2}$ isotherm to provide accurate predictions. This is due to the fact that $\mathrm{CO}_{2}$ readily enters the side pockets along the
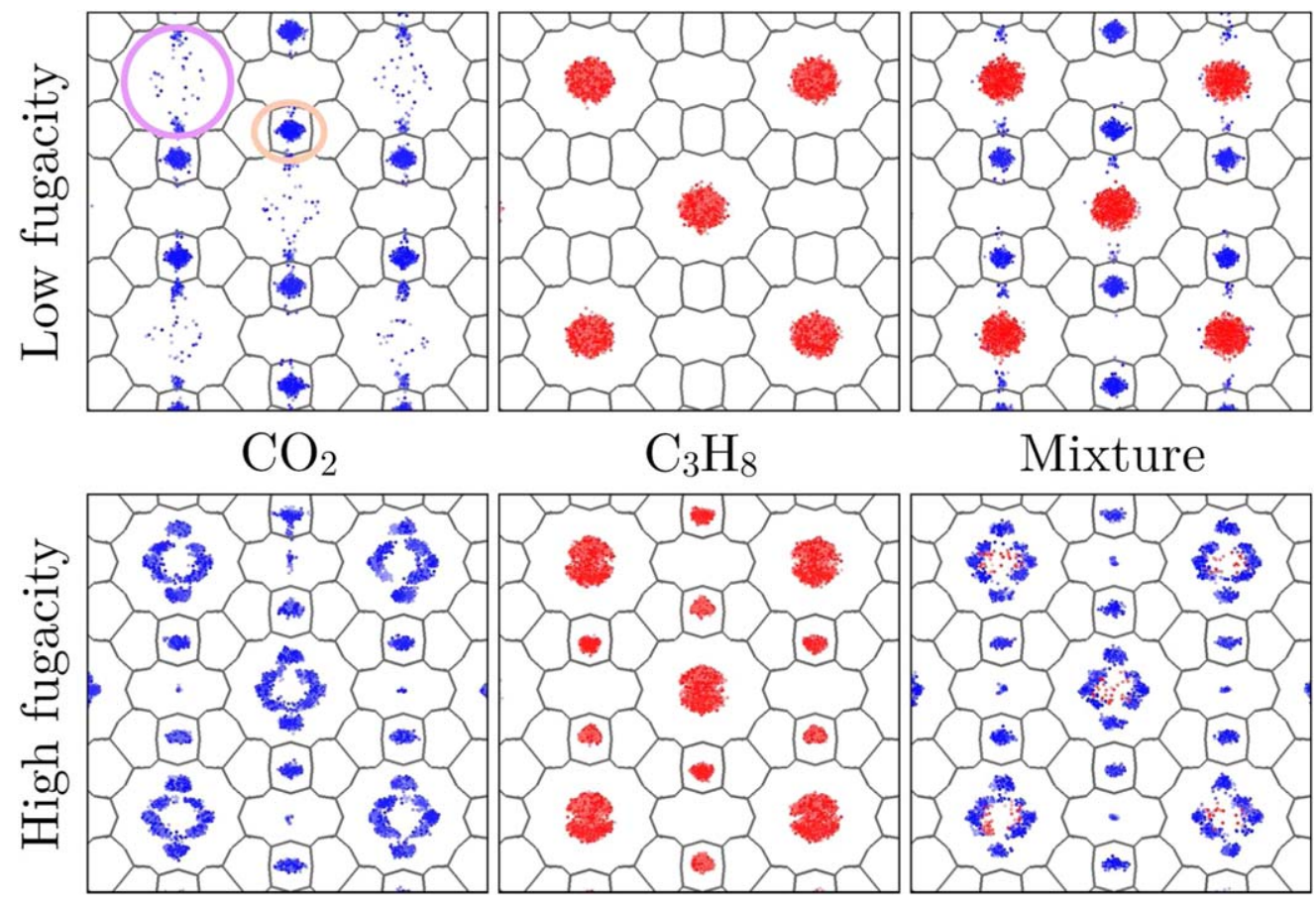

Figure 7. Snapshots of $\mathrm{CO}_{2}$ (blue) and $\mathrm{C}_{3} \mathrm{H}_{8}$ (red) center-of-mass configurations in zeolite MOR during simulation at low $\left(10^{3} \mathrm{~Pa}\right)$ and high $\left(10^{9} \mathrm{~Pa}\right)$ fugacity and $300 \mathrm{~K}$.

Framework silicon and oxygen atoms are indicated in gray, and all views look parallel to the $c$ crystal axis. The centers-of-mass were collected from 10,000 samples during the GCMC simulations. The purple and orange circles in the top left figure show the main channels and side pockets of the material, respectively. While both components can occupy either site, $\mathrm{CO}_{2}$ tends to adsorb in the pockets first, while $\mathrm{C}_{3} \mathrm{H}_{8}$ only enters the pockets at high fugacity. [Color figure can be viewed in the online issue, which is available at wileyonlinelibrary.com.] 

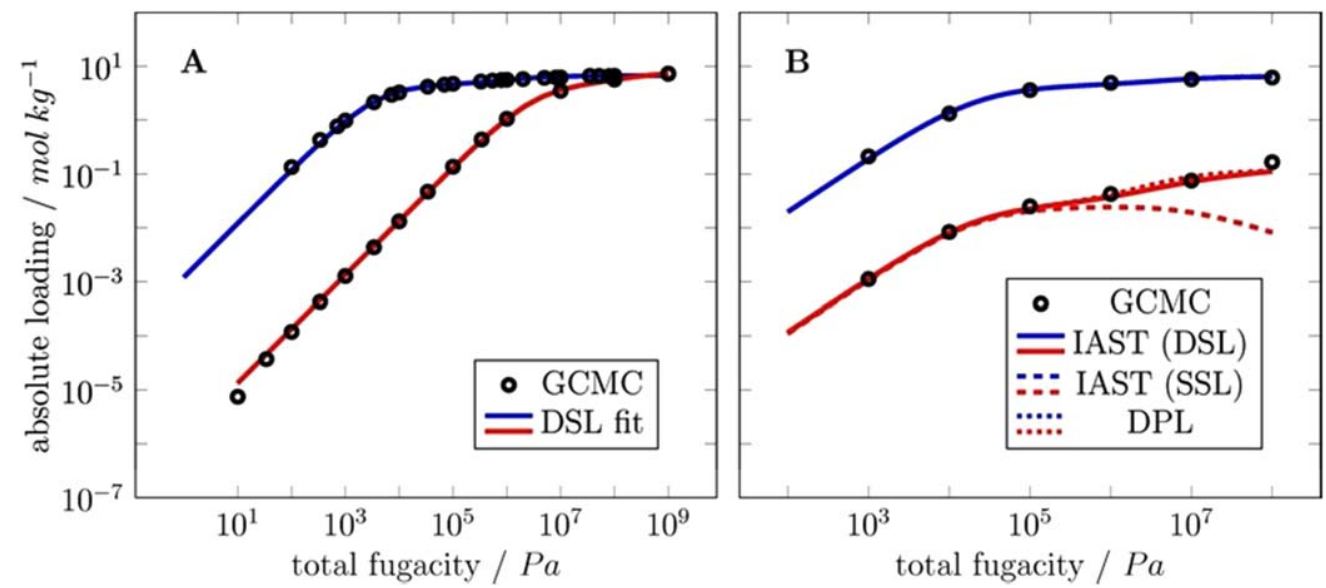

Figure 8. Comparison of isotherms computed via GCMC and different mixture adsorption models in aluminosilicate $\mathrm{NaFAU}(13 \mathrm{X})$ at $300 \mathrm{~K}$.

(A) Pure-component isotherms of $\mathrm{CO}_{2}$ (blue) and $\mathrm{N}_{2}$ (red) with lines indicating the fit of the dual-site Langmuir model (Eq. 10). (B) Mixture adsorption isotherms for a $14 \% \mathrm{CO}_{2}$ (blue), $86 \% \mathrm{~N}_{2}$ mixture. Symbols indicate GCMC results, solid lines represent the SIAST model, and the dashed line represents the traditional IAST model. [Color figure can be viewed in the online issue, which is available at wileyonlinelibrary.com.]

channels of MOR, whereas $\mathrm{C}_{3} \mathrm{H}_{8}$ first adsorbs in the main channels and enters the side pockets at high pressure. This behavior can readily be seen in snapshots of the molecular configurations recorded during the GCMC simulations. As only one molecule can occupy these side pockets at a time, it is appropriate that the loading of the first site in the $\mathrm{CO}_{2}$ isotherm and second site in the $\mathrm{C}_{3} \mathrm{H}_{8}$ isotherm are comparable. The nonideal nature of this system arose solely from this difference in siting behavior and is not a necessarily the result of nonideality in the $\mathrm{CO}_{2}: \mathrm{C}_{3} \mathrm{H}_{8}$ fluid mixture. Unfortunately, the matching of the correct spatial placement of the sites is not easy to ascertain from simply fitting the purecomponent isotherms. The complementary site-matching or perfect negative correlation described by Moon and Tien and Ritter et al., respectively, accounts for this ordering, but requires either additional parameterization or evaluation against experimental data to work. Information about the siting of molecules is a direct result of GCMC simulations and can provide a direct method for determining the correct pairing of sites when applying SIAST with no additional assumptions.

\section{Materials where sites are not isolated}

Another important class of materials for gas separations are aluminosilicate zeolites. The presence of aluminum atoms in the framework creates a charge imbalance that is compensated by the presence of cations in the pores of the material. These cations can be exchanged by washing the materials with solutions of metal salts, and a mixture of different cations can be used to modify the adsorption properties of the materials for a specific purpose. Recent studies on the common type $\mathrm{X}$ and type A zeolites suggest that they may be selective adsorbents for separating mixtures of $\mathrm{CO}_{2}$ and $\mathrm{N}_{2} \cdot{ }^{39-41}$ They typically exhibit a very large selectivity for $\mathrm{CO}_{2}$ over $\mathrm{N}_{2}$ due to the stronger interactions with the larger quadrupole moment of $\mathrm{CO}_{2}$. We simulated adsorption of $\mathrm{CO}_{2}, \mathrm{~N}_{2}$, and a $15 \%: 85 \% \mathrm{CO}_{2}: \mathrm{N}_{2}$ mixture in zeolite $13 \mathrm{X}$ (NaFAU). This material has a Si:Al ratio of approximately 1.24:1. Adsorption of these gases occurs in the supercages of the material, which are connected by $7.4 \AA$ windows. The relatively large distances in these materials suggest that there are not volumes where an adsorbed molecule is segregated from the other pores space and can exclude other molecules. Figure 8A shows the adsorption isotherms for the pure gases at $313 \mathrm{~K}$. Details of the simulation are given in the Supporting Information.

The $\mathrm{CO}_{2}$ isotherm exhibits two-site behavior that can be well correlated using the DSL isotherm. Due to the weak adsorption of $\mathrm{N}_{2}$ at ambient conditions, it is difficult to estimate the saturation loading from typically experimental isotherms. As seen in Figure 8A, it would take on the order of $10^{8} \mathrm{~Pa}$ fugacity to observe saturation loading of $\mathrm{N}_{2}$. In the absence of the required saturation loading, it is often assumed that the saturation loading for one component eventually takes the same value as the other component and a single-site isotherm. This assumption, in and of itself, is not a bad approximation for this system. At the highest fugacity simulated $\left(10^{9} \mathrm{~Pa}\right)$, the loading of $\mathrm{CO}_{2}$ and $\mathrm{N}_{2}$ agree well (Figure 8A). However, it is possible to fit a dual-site model to the $\mathrm{N}_{2}$ data with a high level of confidence. Figure 8B compares the application of traditional IAST using a dualsite model for $\mathrm{CO}_{2}$ and either a single-site or dual-site model for $\mathrm{N}_{2}$. We have also included the dual-process model of Ritter et al. to demonstrate the utility of using competitive models for describing this system.

Figure $8 \mathrm{~B}$ shows that the application of IAST with a dualsite model for both components improves the prediction of mixture isotherms for $\mathrm{N}_{2}$ in $\mathrm{NaX}$ compared to using a single-site isotherm for $\mathrm{N}_{2}$. The $\mathrm{CO}_{2}$ isotherm is not strongly influenced by the choice of isotherm model for $\mathrm{N}_{2}$, reflecting the fact that the stronger adsorption of $\mathrm{CO}_{2}$, reflected by its larger adsorption constants, means that it will always be the preferred molecule to adsorb at a given site. Although it is the more likely component to adsorb at either site, $\mathrm{CO}_{2}$ will tend occupy the strongest site first, as this site represents the lowest free energy for the molecule. It is important that the isotherm for $\mathrm{N}_{2}$ also reflects this choice of adsorption site, even if the preference between the sites is not as strong as 
the other component. When $\mathrm{CO}_{2}$ adsorbs at its lowest free energy site, it will tend to compete with $\mathrm{N}_{2}$ to a lesser extent at the higher free energy site, and consequently the $\mathrm{N}_{2}$ adsorbs to a greater extent than would otherwise be expected. If a single-site isotherm is used to describe $\mathrm{N}_{2}$ adsorption, the predicted mixture contain less nitrogen, because $\mathrm{CO}_{2}$ will always be preferred at a given adsorption site and $\mathrm{N}_{2}$ does not have the option at adsorbing at another site that represents a relatively high free energy for $\mathrm{CO}_{2}$. In contrast to the materials considered in the sections earlier, there is not a spatial segregation of adsorption sites, and both the traditional IAST and the DPL model provide a similar description of adsorption in these materials. Correct modeling of the mixture adsorption requires that each pure component is represented by a model that represents all possible sites where it may adsorb.

\section{Conclusions}

We have evaluated the prediction of adsorption isotherms for mixtures of gases in nanoporous materials using IAST on segregated adsorbed phases. This approach can provide a better description of adsorption in these materials, especially when there are distinct adsorption sites with different saturation behaviors. It is not necessary to directly treat the potential nonideality of the adsorbed mixture, because most of the nonideality is due to the differing preferences for the mixture components for different adsorption sites. We think simplicity of this model makes it sufficiently cheap to calculate for use in the screening of databases of materials for separations or for use in other computational modeling that would benefit from a computationally cheap description of mixture adsorption. High-pressure isotherm data are important to accurately fit saturation loadings. Any model of a pure component used to predict mixture adsorption should describe any multiple-site behavior, even if the preference for different sites is weak. This is especially important when interpreting isotherms where good estimates of the saturation loadings of all components are not available.

\section{Acknowledgments}

J.A.S. was supported by funding from the Advanced Research Projects Agency-Energy (ARPA-E), U.S. Department of Energy. L.-C.L. was supported by the Deutsche Forschungsgemeinschaft (DFG, priority program SPP 1570). J.K. was supported by the U.S. Department of Energy under contract DE-AC02-05CH11231 through the Carbon Capture Simulation Initiative (CCSI). B.S. was supported as part of the Center for Gas Separations Relevant to Clean Energy Technologies, an Energy Frontier Research Center funded by the U.S. Department of Energy, Office of Science, Office of Basic Energy Sciences under Award Number DE-SC0001015. Figures 3, 5, and 7 were made with VMD software support. VMD is developed with NIH support by the Theoretical and Computational Biophysics group at the Beckman Institute, University of Illinois at UrbanaChampaign.

\section{Literature Cited}

1. Keller GE II, Anderson RA, Yon CM. Adsorption. In: Rousseau RW, editor. Handbook of Separation Process Technology. New York: Wiley, 1987:644-696.

2. Myers AL, Prausnitz JM. Thermodynamics of mixed-gas adsorption. AIChE J. 1965;11:121-127.
3. Costa E, Sotelo JL, Calleja G, Marrón C. Adsorption of binary and ternary hydrocarbon gas mixtures on activated carbon: experimental determination and theoretical prediction of the ternary equilibrium data. AIChE J. 1981;27:5-12.

4. Myers AL. Activity coefficients of mixtures adsorbed on heterogeneous surfaces. AIChE J. 1983;29:691-693.

5. Talu O, Zwiebel I. Multicomponent adsorption equilibria of nonideal mixtures. AIChE J. 1986;32:1263-1276.

6. Moon H, Tien C. Adsorption of gas mixtures on adsorbents with heterogeneous surfaces. Chem Eng Sci. 1988;43:2967-2980.

7. Eiden U, Schlünder E. Adsorption equilibria of pure vapors and their binary mixtures on activated carbon. Part I. Single-component equilibria. Chem Eng Process. 1990;28:1-11.

8. Eiden U, Schlünder E. Adsorption equilibria of pure vapors and their binary mixtures on activated carbon. Part II. Multicomponent equilibria. Chem Eng Process. 1990;28:13-22.

9. Chen H, Sholl DS. Examining the accuracy of ideal adsorbed solution theory without curve-fitting using transition matrix Monte Carlo simulations. Langmuir. 2007; 23:6431-6437.

10. Krishna R, van Baten J. Segregation effects in adsorption of $\mathrm{CO}_{2}$-containing mixtures and their consequences for separation selectivities in cage-type zeolites. Sep Purif Technol. 2008;61:414-423.

11. Myers AL. Prediction of adsorption of nonideal mixtures in nanoporous materials. Adsorption. 2005;11:37-42.

12. Valenzuela DP, Myers AL, Talu O, Zwiebel I. Adsorption of gas mixtures: effect of energetic heterogeneity. AIChE J. 1988;34:397402.

13. Cerofolini CF, Rudzinski W. Theoretical principles of singleand mixed-gas adsorption equilibria on heterogenous solid surfaces. In: Rudzinski W, Steele WA, Zgrablich G, editors. Studies in Surface Science and Catalysis. Amsterdam: Elsevier, 1997:1-103.

14. Arnrich S, Kalies G, Bräuer P. About the limits of regularization and the ansatz method. Adsorption. 2011;17:823-831.

15. Ritter JA, Bhadra SJ, Ebner AD. On the use of the dual-process Langmuir model for correlating unary equilibria and predicting mixed-gas adsorption equilibria. Langmuir. 2011;27:4700-4712.

16. Cessford NF, Seaton NA, Düren T. Evaluation of ideal adsorbed solution theory as a tool for the design of metal-organic framework materials. Ind Eng Chem Res. 2012;51:4911-4921.

17. Jee SE, Sholl DS. Carbon dioxide and methane transport in DDR zeolite: insights from molecular simulations into carbon dioxide separations in small pore zeolites. J Am Chem Soc. 2009;131:7896-7904.

18. Van Heest T, Teich-McGoldrick SL, Greathouse JA, Allendorf MD, Sholl DS. Identification of metal-organic framework materials for adsorption separation of rare gases: applicability of ideal adsorbed solution theory (IAST) and effects of inaccessible framework regions. J Phys Chem C. 2012;116:13183-13195.

19. Li JR, Sculley J, Zhou HC. Metal-organic frameworks for separations. Chem Rev. 2012;112:869-932.

20. Pophale R, Cheeseman PA, Deem MW. A database of new zeolitelike materials. Phys Chem Chem Phys. 2011;13:12407-12412.

21. Wilmer CE, Leaf M, Lee CY, Farha OK, Hauser BG, Hupp JT, Snurr RQ. Large-scale screening of hypothetical metal-organic frameworks. Nat Chem. 2012;4:83-89.

22. DAlessandro DM, Smit B, Long JR. Carbon dioxide capture: prospects for new materials. Angew Chem Int Ed. 2010;49:6058-6082.

23. Sumida K, Rogow DL, Mason JA, McDonald TM, Bloch ED, Herm ZR, Bae TH, Long JR. Carbon dioxide capture in metal-organic frameworks. Chem Rev. 2012;112:724-781.

24. Suh MP, Park HJ, Prasad TK, Lim DW. Hydrogen storage in metalorganic frameworks. Chem Rev. 2012;112:782-835.

25. Lin LC, Berger AH, Martin RL, Kim J, Swisher JA, Jariwala K, Rycroft CH, Bhown AS, Deem MW, Haranczyk M, Smit B. In silico screening of carbon-capture materials. Nat Mater. 2012;11:633-641.

26. Frenkel D, Smit B. Understanding Molecular Simulation: From Algorithms to Applications, 2nd ed. San Diego: Academic Press, 2002.

27. Smit B. Grand canonical Monte Carlo simulations of chain molecules: adsorption isotherms of alkanes in zeolites. Mol Phys. 1995;85:153-172.

28. Walton KS, Millward AR, Dubbeldam D, Frost H, Low JJ, Yaghi OM, Snurr RQ. Understanding inflections and steps in carbon dioxide adsorption isotherms in metal-organic frameworks. J Am Chem Soc. 2008; 130:406-407.

29. McDaniel JG, Yu K, Schmidt JR. Ab initio, physically motivated force fields for $\mathrm{CO}_{2}$ adsorption in zeolitic imidazolate frameworks. J Phys Chem C. 2012;116:1892-1903. 
30. Scholl S, Schachtl M, Sievers W, Schweighart P, Mersmann A. Calculation methods for multicomponent adsorption equilibria. Chem Eng Technol. 1991;14:311-324.

31. Richter E, Wilfried S, Myers AL. Effect of adsorption equation on prediction of multicomponent adsorption equilibria by the ideal adsorbed solution theory. Chem Eng Sci. 1989;44:1609-1616.

32. Sievers W, Mersmann A. Single and multicomponent adsorption equilibria of carbon dioxide, nitrogen, carbon monoxide and methane in hydrogen purification processes. Chem Eng Technol. $1994 ; 17: 325-337$

33. Hu X, Do DD. Comparing various multicomponent adsorption equilibrium models. AIChE J. 1995;41:1585-1592.

34. Krishna R, Calero S, Smit B. Investigation of entropy effects during sorption of mixtures of alkanes in MFI zeolite. Chem Eng J. 2002;88:81-94.

35. Gumma S, Talu O. Net adsorption: a thermodynamic framework for supercritical gas adsorption and storage in porous solids. Langmuir. 2010;26:17013-17023.

36. García-Pérez E, Parra J, Ania C, García-Sánchez A, van Baten J, Krishna R, Dubbeldam D, Calero S. A computational study of $\mathrm{CO}_{2}$, $\mathrm{N}_{2}$, and $\mathrm{CH}_{4}$ adsorption in zeolites. Adsorption. 2007;13:469-476.
37. Dubbeldam D, Calero S, Vlugt TJH, Krishna R, Maesen TLM, Smit B. United atom force field for alkanes in nanoporous materials. J Phys Chem B. 2004;108:12301-12313.

38. García-Sánchez A, Ania CO, Parra JB, Dubbeldam D, Vlugt TJH, Krishna R, Calero S. Transferable force field for carbon dioxide adsorption in zeolites. J Phys Chem C. 2009;113:8814-8820.

39. Cavenati S, Grande CA, Rodrigues AE. Adsorption equilibrium of methane, carbon dioxide, and nitrogen on zeolite $13 \mathrm{X}$ at high pressures. J Chem Eng Data. 2004;49:1095-1101.

40. Wang Y, LeVan MD. Adsorption equilibrium of binary mixtures of carbon dioxide and water vapor on zeolites $5 \mathrm{~A}$ and $13 \mathrm{X}$. J Chem Eng Data. 2010;55:3189-3195.

41. Bae TH, Hudson MR, Mason JA, Queen WL, Dutton JJ, Sumida K, Micklash KJ, Kaye SS, Brown CM, Long JR. Evaluation of cationexchanged zeolite adsorbents for post-combustion carbon dioxide capture. Energy Environ Sci. 2013;6:128-138.

Manuscript received Oct. 9, 2012, and revision received Jan. 24, 2013 